\title{
Ensaio mecânico da resistência ao impacto do cimento ósseo puro e associado a duas drogas anestésicas locais*
}

\author{
Mechanical essay of impact resistance of acrylic bone cement used \\ singly or the cement in combination with two local anesthetic drugs
}

\author{
Vincenzo Giordano', Henrique Rios ${ }^{2}$, Marcos Moreirão3 ${ }^{3}$ Marcos Giordano4, \\ Ney Pecegueiro do Amaral ${ }^{5}$, Alexandre Pallottino ${ }^{6}$, Silvio de Oliveira ${ }^{2}$
}

\section{RESUMO}

Objetivo: Investigar o comportamento mecânico da combinação de anestésico local e cimento ósseo in vitro. Métodos: Foram comparados dois cimentos ortopédicos (Simplex $^{\circledR}$ e Biomecânica $\left.{ }^{\circledR}\right)$ e duas drogas anestésicas locais de largo uso na clínica anestesiológica (lidocaína e bupivacaína). Os anestésicos utilizados estavam em pó. Elaboraramse seis grupos de investigação, baseados na combinação ou não das drogas. Nos grupos em que o polímero foi combi-

* Investigação realizada no Serviço de Ortopedia e Traumatologia Prof. Nova Monteiro - Hospital Municipal Miguel Couto (SOT-HMMC), no Departamento de Biomecânica - Instituto Petroflex Rio de Janeiro, e no Laboratório da Souza Cruz, Rio de Janeiro (RJ), Brasil.

1. Coordenador da Residência Médica do Serviço de Ortopedia e Traumatologia Prof. Nova Monteiro - Hospital Municipal Miguel Couto - SOT HMMC - Rio de Janeiro (RJ), Brasil.

2. Médico Ex-Residente do SOT-HMMC. Informação atual ex não deve ser usado.

3. Residente (R3) do Serviço de Ortopedia e Traumatologia Prof. Nova Monteiro - Hospital Municipal Miguel Couto - SOT - HMMC - Rio de Janeiro (RJ), Brasil.

4. Coordenador da Residência Médica do Hospital de Força Aérea do Galeão - HFAG - Rio de Janeiro (RJ), Brasil. Verificar se é o correto.

5. Chefe do Serviço de Ortopedia e Traumatologia Prof. Nova Monteiro Hospital Municipal Miguel Couto - SOT - HMMC - Rio de Janeiro (RJ), Brasil.

6. Médico Assistente do Serviço de Ortopedia e Traumatologia Prof. Nova Monteiro - Hospital Municipal Miguel Couto - SOT - HMMC - Rio de Janeiro (RJ), Brasil.

Endereço para correspondência: Vincenzo Giordano, Rua Aristides Espínola, 11/301, Leblon - 22440-050 - Rio de Janeiro (RJ) - Brasil. Tel./fax: (+55 21) 2274-6830. E-mail: sot.hmmc@terra.com.br

Recebido em 30/11/06. Aprovado para publicação em 18/7/07.

Copyright RBO2007 nado à medicação, a mistura consistiu de $40 \mathrm{~g}$ de polimetilmetacrilato com $2 \mathrm{~g}$ de anestésico local. Foram confeccionados 60 corpos de prova prismáticos, medindo $5 \times 120 \times$ $30 \mathrm{~mm}(\mathrm{n}=30)$ e $5 \times 60 \times 30 \mathrm{~mm}(\mathrm{n}=30)$. Os corpos de prova foram testados mecanicamente em máquina universal. Foram realizados ensaios mecânicos de resistência ao impacto direto. Foi realizada análise estatística para verificar o efeito do cimento (Simplex ${ }^{\circledR}$ e Biomecânica ${ }^{\circledR}$ ) e da medicação (lidocaína e bupivacaína) na resistência do corpo de prova, com $\alpha=5 \%$. Resultados: Observou-se que existe influência significativa da medicação na resistência do corpo de prova $(p=0,0001)$. Pelo teste de comparações múltiplas de Tukey, identificou-se, ao nível de 5\%, que a mistura com bupivacaína apresentou resistência significativamente maior do que com a lidocaína e com o polímero puro. Não existe diferença significativa na resistência entre a lidocaína e o polímero puro. Existe influência significativa do cimento na resistência do corpo de prova $(p=$ 0,015). Mostrou-se que o cimento Simplex ${ }^{\circledR}$ apresentou resistência significativamente maior do que o Biomecânica ${ }^{\circledR}$. Existe influência significativa da interação cimento-medicação na resistência do corpo de prova $(p=0,035)$. A análise dos contrastes mostrou que o cimento Simplex ${ }^{\circledR}$ apresentou resistência significativamente maior do que o Biomecânica $^{\circledR}$ apenas quando sem adição da medicação ( $p=$ $0,002)$. Não existe diferença significativa na resistência entre os cimentos Simplex ${ }^{\circledR}$ e Biomecânica ${ }^{\circledR}$ para as medicações lidocaína $(p=0,13)$ e bupivacaína $(p=0,63)$. No cimento Simplex $^{\circledR}$, a associação com a bupivacaína apresentou resistência significativamente maior do que com a lidocaína e o polímero puro $(\mathrm{p}=\mathbf{0 , 0 0 1} \mathrm{e} \mathbf{p}=\mathbf{0 , 0 1 2}$, respectivamente). Não existe diferença significativa na resistência entre a li- 
docaína e o polímero puro para o cimento Simplex ${ }^{\circledR}(\mathrm{p}=$ 0,39). No cimento Biomecânica ${ }^{\circledR}$, a associação com a bupivacaína apresentou resistência significativamente maior do que com a lidocaína e o polímero puro $(\mathbf{p}=0,0001$ e $\mathbf{p}=$ 0,0001 , respectivamente). Não existe diferença significativa na resistência entre a lidocaína e o polímero puro para o cimento Biomecânica ${ }^{\circledR}(p=0,37)$. Conclusão: Nas condições estudadas, não há redução significativa da resistência ao impacto na combinação de cimento ortopédico com anestésicos locais.

Descritores - Cimentos para ossos; Polimetil metacrilato; Lidocaína; Bupivacaína

\section{ABSTRACT}

Objective: To investigate the mechanical behavior of cement-local anesthetic combinations in vitro. Methods: Two bone cements were tested (Simplex ${ }^{\circledR}$ and Biomecânica ${ }^{\circledR}$ ) with two anesthetic drugs (lidocaine and bupivacaine). Anesthetic drugs were added in powder form. The authors compared six groups based on the association between the cement and the drugs. Two grams of anesthetic were mixed with $40 \mathrm{~g}$ of acrylic cement powder. 60 prismatic molds were made, measuring $5 \times 120 \times 30 \mathrm{~mm}(n=30)$ and $5 \times 60 \times 30 \mathrm{~mm}(n=$ 30). The molds were tested on a pendulum impact resistance apparatus. Statistical analysis was performed to verify the effect of bone cement (Simplex ${ }^{\circledR}$ and Biomecânica ${ }^{\circledR}$ ) and the medication (lidocaine, bupivacaine, and no combination) on the strength of the molds, with a level of significance $\alpha=5 \%$. Results: Statistical comparison showed a significant influence of the medication on bone strength ( $p=0.0001$ ). Tukey multiple comparison test demonstrated better strength with bupivacaine. Conclusion: Cement-bone combination does not harm the strength of the cement itself.

Keywords - Bone cements; Polymethyl methacrylate; Lidocaine; Bupivacaine

\section{INTRODUÇÃO}

A utilização de cimento ósseo em artroplastias revolucionou o conceito de fixação do implante ao osso ${ }^{(1)}$. Sua principal função é preencher o espaço existente entre a prótese e o osso hospedeiro, atuando como interface elástica capaz de transmitir cargas de uma superfície a outra ${ }^{(2-3)}$. Essa função de distribuição de estresse é fundamental para que se criem condições de estabilidade do implante a longo tempo, aumentando a durabilidade e o sucesso do procedimento cirúrgico ${ }^{(1-4)}$.
Mais recentemente, outras funções foram atribuídas ao cimento ortopédico. Uma das mais interessantes tem sido seu uso como veículo de liberação local de drogas tradicionalmente utilizadas por outras vias de administração. O conceito de liberação local de drogas em alta concentração permite o emprego de doses maiores do fármaco sem as complicações secundárias inerentes ao seu uso de forma sistêmica ${ }^{(5)}$. Buchholz et al realizaram as primeiras tentativas de adicionar antibióticos ao cimento ósseo como medida adjuvante no manejo de artroplastias de quadril infectadas ${ }^{(6)}$. Atualmente, cerca de $90 \%$ dos cirurgiões ortopédicos norte-americanos usam esse veículo de liberação local de antibiótico, existindo cinco tipos de cimento ortopédico associado a antimicrobianos já aprovados pelo FDA (Food and Drug Administration) ${ }^{(5,7)}$.

As vantagens demonstradas com a associação de antibiótico ao cimento ósseo foram recentemente estendidas. Bond $e t$ al adicionaram medicações anestésicas locais a esse polímero e realizaram investigação in vitro da liberação dessa droga durante 72 horas $^{(8)}$. Em teoria, conforme citado pelos próprios autores, a obtenção de níveis locais elevados dessas drogas reduziria de forma dramática o quadro álgico de pacientes submetidos a procedimentos de substituição articular, especialmente nas primeiras horas após a cirurgia ${ }^{(8)}$. Além disso, inúmeras outras vantagens podem advir do sucesso da combinação de anestésicos locais e cimento ortopédico. Dentre essas, destacamos a redução dos níveis sistêmicos de drogas opióides e analgésicas, maior cooperação do paciente com o processo de reabilitação nos primeiros dias pós-artroplastia, mobilização mais precoce (diminuindo os riscos inerentes ao paciente idoso acamado) e maior satisfação (e aceitação) com $\mathrm{o}$ ato operatório em si.

Apesar disso, as propriedades mecânicas do cimento ortopédico são afetadas pela associação com outras substâncias ${ }^{2-}$ 3,9). Sabe-se do aprendizado com drogas antimicrobianas que antibióticos líquidos produzem maior redução da resistência do polímero às forças de compressão do que sua apresentação em pó (cristalina) ${ }^{(9-10)}$. No entanto, não foi demonstrada redução significativa da resistência na combinação de cimento ortopédico com antimicrobianos, de forma que apresentações comerciais dessa mistura estão disponíveis no meio ortopédico.

Com relação ao conjunto cimento ortopédico-drogas anestésicas locais, pouco ou nada se sabe até o momento. Até onde alcança nosso conhecimento, estudos mecânicos com essa associação não foram realizados.

O objetivo dos autores foi investigar o comportamento mecânico ao impacto de corpos de prova confeccionados com a combinação de anestésico local e cimento ósseo. 


\section{MÉTODOS}

Foram comparados dois cimentos ortopédicos $\left(\right.$ Simplex $^{\circledR} \mathrm{e}$ Biomecânica ${ }^{\circledR}$ ) e duas drogas anestésicas locais de largo uso na clínica anestesiológica (lidocaína e bupivacaína). Os anestésicos utilizados estavam em pó. Foram confeccionados 60 corpos de prova prismáticos, medindo $5 \times 120 \times 30 \mathrm{~mm}(\mathrm{n}=$ $30)$ e $5 \times 60 \times 30 \mathrm{~mm}(\mathrm{n}=30)$, sempre pelo mesmo pesquisador. Elaboraram-se seis grupos de investigação, baseados na combinação ou não das drogas: Simplex ${ }^{\circledR}$ puro $(\mathrm{n}=10)$, Simplex $^{\circledR}+$ bupivacaína $(\mathrm{n}=10)$, Simplex ${ }^{\circledR}+$ lidocaína $(\mathrm{n}=10)$, Biomecânica $^{\circledR}$ puro $(\mathrm{n}=10)$, Biomecânica ${ }^{\circledR}+$ bupivacaína ( $\mathrm{n}$ $=10)$, Biomecânica ${ }^{\circledR}+$ lidocaína $(\mathrm{n}=10)$. Nos grupos em que o polímero foi combinado à medicação, a mistura consistiu de $40 \mathrm{~g}$ de polimetilmetacrilato com $2 \mathrm{~g}$ de anestésico local. A mistura do cimento, associado ou não à droga testada, seguiu
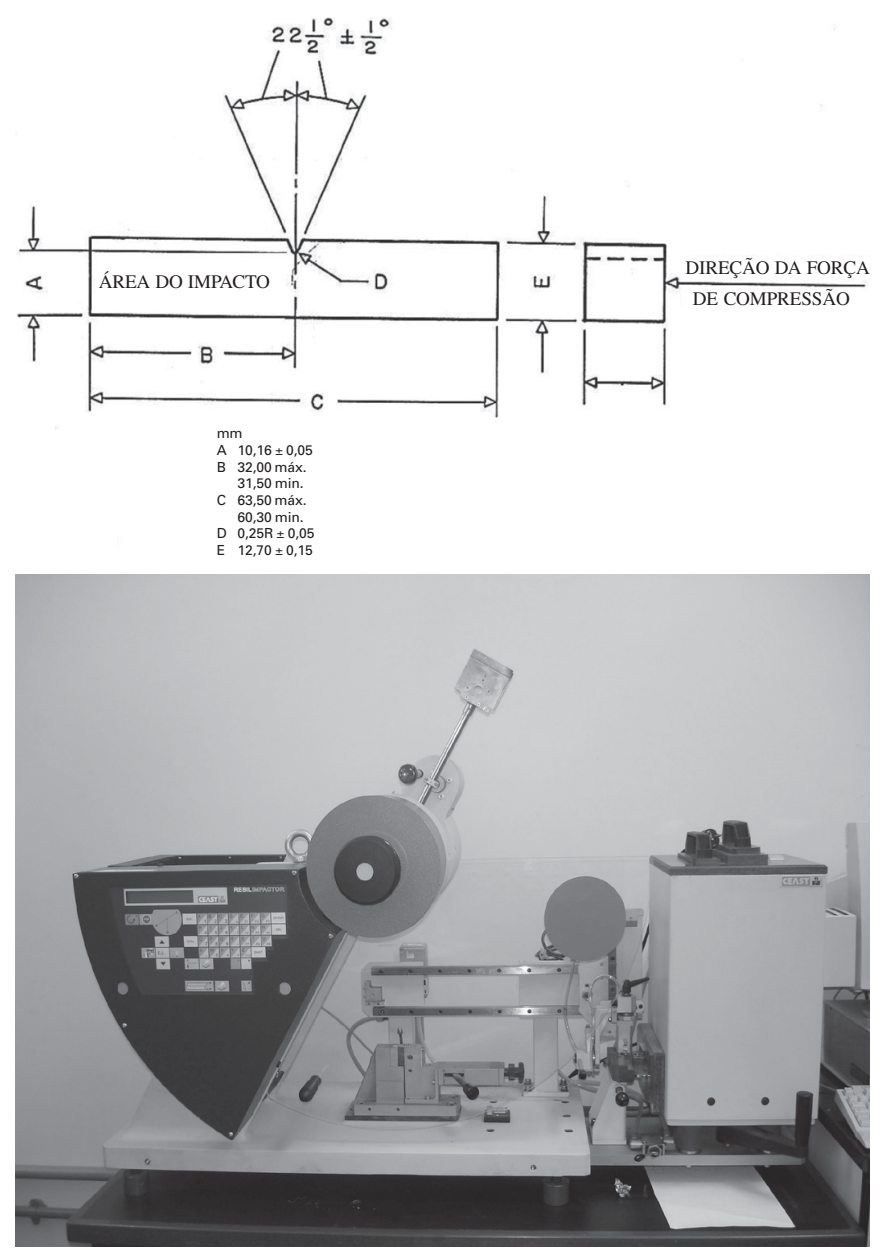

Figura 1 - Máquina universal de testes utilizada para realização de testes de impacto. Acima, vê-se representação esquemática do tipo de teste que está sendo realizado. Abaixo, observa-se a máquina montada para realização de um dos testes mecânicos. protocolo descrito por Bond et al ${ }^{(8)}$. Após a confecção do corpo de prova, este foi identificado, protegido individualmente em plástico-bolha e acondicionado em caixa apropriada até o momento do teste mecânico.

Os corpos de prova foram testados mecanicamente em máquina universal CEAST Resil Impactor. Foram realizados ensaios mecânicos para determinação de resistência de plásticos ao impacto de pêndulo Izod (Izod pendulum impact resistance of plastics) (figura 1). Durante os testes, a resistência do corpo de prova é avaliada pelo impacto de um pêndulo tipomartelo projetado de uma altura-padrão.

Foi realizada análise estatística para verificar o efeito do cimento (Simplex ${ }^{\circledR}$ e Biomecânica ${ }^{\circledR}$ ) e da medicação (lidocaína e bupivacaína) na resistência do corpo de prova utilizandose análise de variância para dois fatores (ANOVA two-way). O teste de comparações múltiplas de Tukey foi aplicado para identificar quais os cimentos (ou medicações) que diferem entre si. As comparações múltiplas da interação foram testadas pelos contrastes do modelo linear generalizado, processado pelo procedimento GLM do software $S A S^{\circledR}$. A resistência foi analisada pelo seu logaritmo natural, pois a mesma não apresentou distribuição normal (gaussiana) devido à falta de simetria. $\mathrm{O}$ critério de determinação de significância adotado foi o nível de $5 \%$.

\section{RESULTADOS}

\section{Análise descritiva geral}

A tabela 1 fornece a média, desvio-padrão (DP), mediana, mínimo e máximo da resistência (joules) para o total do cimento e total da medicação.

\section{TABELA 1}

Análise descritiva da resistência para o total do cimento e medicação

\begin{tabular}{lllllll}
\hline Resistência & N & Média & DP & \multicolumn{3}{c}{ Mediana Mínimo Máximo } \\
\hline Simplex $^{\circledR}$ & 30 & 0,2023 & 0,0468 & 0,1905 & 0,1490 & 0,3320 \\
Biomecânica $^{\circledR}$ & 30 & 0,1839 & 0,0463 & 0,1615 & 0,1440 & 0,3490 \\
Lidocaína $^{20}$ & 0,1714 & 0,0205 & 0,1660 & 0,1490 & 0,2380 \\
Bupivacaína & 20 & 0,2343 & 0,0451 & 0,2260 & 0,1890 & 0,3490 \\
Puro & 20 & 0,1738 & 0,0418 & 0,1590 & 0,1440 & 0,3250
\end{tabular}

Fonte: SOT-HMMC, 2006

DP - desvio-padrão

As tabelas 2 e 3 fornecem a média, desvio-padrão (DP), mediana, mínimo e máximo da resistência (joules), segundo a medicação para o cimento Simplex ${ }^{\circledR}$ e Biomecânica ${ }^{\circledR}$, respectivamente. 
TABELA 2

Análise descritiva da resistência segundo a medicação para o cimento Simplex ${ }^{\circledR}$

\begin{tabular}{lllllll} 
Medicação & N Média & DP & \multicolumn{3}{c}{ Mediana Mínimo Máximo } \\
\hline Lidocaína & 10 & 0,1808 & 0,0247 & 0,1755 & 0,1490 & 0,2380 \\
Bupivacaína & 10 & 0,2309 & 0,0484 & 0,2150 & 0,1890 & 0,3320 \\
Puro & 10 & 0,1952 & 0,0513 & 0,1770 & 0,1490 & 0,3250
\end{tabular}

Fonte: SOT-HMMC, 2006

DP - desvio-padrão

TABELA 3

Análise descritiva da resistência segundo a medicação para o cimento Biomecânica ${ }^{\circledR}$

\begin{tabular}{lllllll}
\hline Medicação & N & Média & DP & \multicolumn{3}{c}{ Mediana Mínimo Máximo } \\
\hline Lidocaína & 10 & 0,1619 & 0,0090 & 0,1615 & 0,1490 & 0,1790 \\
Bupivacaína & 10 & 0,2376 & 0,0439 & 0,2345 & 0,1990 & 0,3490 \\
Puro & 10 & 0,1523 & 0,0059 & 0,1520 & 0,1440 & 0,1610
\end{tabular}

Fonte: SOT-HMMC, 2006

DP - desvio-padrão

Para verificar o efeito do cimento (Simplex ${ }^{\circledR}$ e Biomecâni$c a^{\circledR}$ ), da medicação (lidocaína e bupivacaína) e da interação cimento-medicação na resistência do corpo de prova, foi realizada a análise de variância para dois fatores (ANOVA twoway). A tabela 4 fornece o resultado da análise de variância para dois fatores para o logaritmo da resistência. Foi utilizado o logaritmo natural da resistência, pois esta não apresentou distribuição normal (gaussiana) devido à falta de simetria.
TABELA 4

Resultado da análise de variância para o logaritmo da resistência

\begin{tabular}{lccccc}
\hline \multicolumn{5}{c}{ Variável dependente: logaritmo (LN) da resistência } \\
\hline \multicolumn{1}{c}{ Origem } & df & SS & MS & F & Pr $>$ F \\
\hline \multirow{2}{*}{ Medicação } & 2 & 1,2291 & 0,6145 & 26,8 & 0,0001 \\
Cimento & 1 & 0,1443 & 0,1443 & 6,3 & 0,015 \\
Cimento-medicação & 2 & 0,1645 & 0,0822 & 3,6 & 0,035 \\
\hline
\end{tabular}

Fonte: SOT-HMMC, 2006

Legendas: $\mathrm{df}$ - graus de liberdade

SS - soma dos quadrados da ANOVA

$\mathrm{F}$ - estatística $\mathrm{F}$

$\mathrm{Pr}>\mathrm{F}$ - valor de $\mathrm{p}$

MS - quadrado médio

A tabela 5 fornece o resultado da análise dos contrastes para o logaritmo da resistência (LN resistência).

Observou-se que existe influência significativa da medicação na resistência do corpo de prova $(\mathrm{p}=0,0001)$. Pelo teste de comparações múltiplas de Tukey, identificou-se, ao nível de 5\%, que a mistura com bupivacaína apresentou resistência significativamente maior do que com a lidocaína e com o polímero puro. Não existe diferença significativa na resistência entre a lidocaína e o polímero puro. Existe influência significativa do cimento na resistência do corpo de prova $(\mathrm{p}=0,015)$. Mostrou-se que o cimento Simplex ${ }^{\circledR}$ apresentou resistência significativamente maior do que o Biomecânica ${ }^{\circledR}$. Existe influência significativa da interação cimento-medicação na resistência do corpo de prova $(\mathrm{p}=0,035)$.

TABELA 5

Resultado da análise dos contrastes

Variável dependente: $L N$ resistência

\begin{tabular}{lccccc}
\hline \multicolumn{1}{c}{ Contraste } & df & SS & MS & F & Pr $>$ F \\
\hline Simplex $^{\circledR}$ versus Biomecânica ${ }^{\circledR}$-Lidocaína & 1 & 0,0541 & 0,0541 & 2,4 & 0,13 \\
${\text { Simplex }{ }^{\circledR} \text { versus Biomecânica }{ }^{\circledR} \text {-Bupivacaína }}$ Simplex $^{\circledR}$ versus Biomecânica ${ }^{\circledR}$-Puro & 1 & 0,0055 & 0,0055 & 0,2 & 0,63 \\
& 1 & 0,2493 & 0,2493 & 10,9 & 0,002 \\
\hline Lidocaína versus Bupivacaína-Simplex $^{\circledR}$ & 1 & 0,2754 & 0,2754 & 12,0 & 0,001 \\
Lidocaína versus Puro-Simplex $^{\circledR}$ & 1 & 0,0173 & 0,0173 & 0,8 & 0,39 \\
Bupivacaína versus Puro-Simplex $^{\circledR}$ & 1 & 0,1546 & 0,1546 & 6,7 & 0,012 \\
\hline Lidocaína versus Bupivacaína-Biomecânica $^{\circledR}$ & 1 & 0,6910 & 0,6910 & 30,1 & 0,0001 \\
Lidocaína versus Puro-Biomecânica $^{\circledR}$ & 1 & 0,0183 & 0,0183 & 0,8 & 0,37 \\
Bupivacaína versus Puro-Biomecânica $^{\circledR}$ & 1 & 0,9338 & 0,9338 & 40,7 & 0,0001 \\
\hline
\end{tabular}

Fonte: SOT-HMMC, 2006

Legendas: $\mathrm{df}$ - graus de liberdade

SS - soma dos quadrados da ANOVA

$\mathrm{F}$ - estatística $\mathrm{F}$

$\mathrm{Pr}>\mathrm{F}$ - valor de $\mathrm{p}$

MS - quadrado médio 
A análise dos contrastes mostrou que o cimento Simplex ${ }^{\circledR}$ apresentou resistência significativamente maior do que o Biomecânica ${ }^{\circledR}$ apenas quando sem a adição da medicação $(\mathrm{p}=$ $0,002)$. Não existe diferença significativa na resistência entre os cimentos Simplex ${ }^{\circledR}$ e Biomecânica ${ }^{\circledR}$ para as medicações lidocaína $(\mathrm{p}=0,13)$ e bupivacaína $(\mathrm{p}=0,63)$. No cimento Simplex ${ }^{\circledR}$, a bupivacaína apresentou resistência significativamente maior do que a lidocaína e o polímero puro $(\mathrm{p}=0,001$ e $p=0,012$, respectivamente). Não existe diferença significativa na resistência entre a lidocaína e o polímero puro para o cimento Simplex ${ }^{\circledR}(\mathrm{p}=0,39)$. No cimento Biomecânica ${ }^{\circledR}$, a bupivacaína apresentou resistência significativamente maior do que a lidocaína e o polímero puro $(\mathrm{p}=0,0001 \mathrm{e} \mathrm{p}=0,0001$, respectivamente). Não existe diferença significativa na resistência entre a lidocaína e o polímero puro para o cimento Biomecânica ${ }^{\circledR}(\mathrm{p}=0,37)$.

\section{DISCUSSÃO}

A utilização de cimento ortopédico como veículo de liberação local de diversas drogas vem ganhando cada vez maior dimensão. Estudos recentes expandiram suas possibilidades de combinação além dos antimicrobianos, com drogas antiinflamatórias não-esteróides e agentes antineoplásicos ${ }^{(11-12)}$. Recentemente, Bond et al propuseram seu uso associado a anestésico local ${ }^{(8)}$. Esses autores conduziram estudo de dispersão de três drogas anestésicas (lidocaína, bupivacaína e prilocaína) misturadas ao cimento ósseo. Durante 72 horas, foi quantificada a liberação dessas substâncias em meio salino. Bond et al observaram que a prilocaína foi liberada mais rapidamente e a bupivacaína, mais lentamente ${ }^{(8)}$. Embora outros estudos sejam necessários para que seja possível determinar corretamente a dose ideal de cada droga de uso potencial, os ensinamentos de Bond et al, abrem inúmeras perspectivas em termos de manejo de dor pós-operatória em artroplastia ${ }^{(8)}$.

Atualmente, diversos protocolos são empregados no controle do quadro álgico pós-artroplastia, indo desde morfina intratecal até analgesia controlada pelo paciente. Um dos grandes problemas relacionados a isso é o risco elevado de toxicidade em pacientes expostos a altas concentrações sistêmicas de medicações analgésicas. A associação do anestésico local ao cimento ósseo torna possível reduzir a administração de drogas por outras vias, elevando a capacidade de analgesia por liberação local em altas doses.

Sabe-se que as propriedades mecânicas de um polímero são definidas, em geral, pela realização de uma série de testes in vitro. No entanto, para que a combinação cimento-anestésico possa ser utilizada in vivo, estudos de resistência mecâni- ca da mistura devem ser realizados. No presente experimento, investigamos a resistência ao impacto de corpos de prova produzidos com e sem a adição de drogas anestésicas. Seguimos exatamente o modelo criado por Bond et al, exceto pela prilocaína $^{(8)}$. Nossos resultados demonstram vários pontos bastante interessantes e que suscitam alguns aspectos de discussão.

A associação com as drogas melhora a resistência do cimento. Comparativamente, quando o polímero está sem adição de medicações, há diferença estatisticamente significativa entre os dois grupos. No entanto, após sua associação com algum anestésico local, ocorre melhora substancial da resistência do cimento ósseo. Embora pareça difícil de compreender, acreditamos que tal fato se deva ao uso de composições de anestésico em pó. Ger et al observaram que antibióticos líquidos reduzem a resistência do cimento ósseo quando comparados ao pó ${ }^{(10)}$. Da mesma forma, tem sido observado que a polimerização do cimento ortopédico é francamente influenciada pela relação entre pó e líquido ${ }^{(2)}$. Cremos que a adição de mais pó à mistura trouxe aumento de resistência ao conjunto simplesmente por haver mais massa física.

Nosso experimento dá mais um passo para que futuras investigações possam ser conduzidas em termos de avaliação da real analgesia (e suas vantagens teóricas) após procedimento de substituição articular utilizando cimento ósseo. Mostramos que a combinação cimento-anestésico local não reduz a resistência ao impacto do conjunto. Apesar de a bupivacaína ter apresentado resistência estatisticamente superior à da lidocaína, ambas melhoraram as propriedades mecânicas do cimento. Diferentes testes mecânicos devem ser realizados para que sua utilização in vivo possa finalmente ser investigada.

\section{CONCLUSÃO}

Nas condições estudadas, não há redução significativa da resistência ao impacto na combinação de cimento ortopédico com anestésicos locais.

\section{REFERÊNCIAS}

1. Charnley J. Anchorage of the femoral head prosthesis of the shaft of the femur. J Bone Joint Surg Br. 1960;42-B:28-30.

2. Kuehn KD, Ege W, Gopp U. Acrylic bone cements: composition and properties. Orthop Clin North Am. 2005;36(1):17-28, v.

3. Kuehn KD, Ege W, Gopp U. Acrylic bone cements: mechanical and physical properties. Orthop Clin North Am. 2005;36(1):29-39, v-vi. Review.

4. Dorr LD, Wan Z, Gruen T. Functional results in total hip replacement in patients 65 years and older. Clin Orthop Relat Res. 1997;(336):143-51.

5. Nelson CL. The current status of material used for depot delivery of drugs. Clin Orthop Relat Res. 2004;(427):72-8. 
6. Buchholz HW, Elson RA, Engelbrecht E, Lodenkämper H, Röttger J, Siegel A. Management of deep infection of total hip replacement. J Bone Joint Surg Br. 1981;63-B(3):342-53.

7. Heck D, Rosenberg A, Schink-Ascani M, Garbus S, Kiewitt T. Use of antibiotic-impregnated cement during hip and knee arthroplasty in the United States. J Arthroplasty. 1995;10(4):470-5.

8. Bond DM, Rudan J, Kobus SM, Adams MA. Depot local anesthetic in polymethylmethacrylate bone cement: a preliminary study. Clin Orthop Relat Res. 2004;(418):242-5.
9. Armstrong MS, Spencer RF, Cunningham JL, Gheduzzi S, Miles AW, Learmonth ID. Mechanical characteristics of antibiotic-laden bone cement. Acta Orthop Scand. 2002;73(6):688-90.

10. Ger E, Dall D, Miles T, Forder A. Bone cement and antibiotics. S Afr Med J. 1977;51(9):276-9.

11. Corry D, Moran J. Assessment of acrylic bone cement as a local delivery vehicle for the application of non-steroidal anti-inflammatory drugs. Biomaterials. 1998;19(14):1295-301.

12. Healey JH, Shannon F, Boland P, DiResta GR. PMMA to stabilize bone and deliver antineoplastic and antiresorptive agents. Clin Orthop Relat Res. 2003;(415 Suppl):S263-75. 